LAW-DMR 2021

\title{
The Joint 15th Linguistic Annotation Workshop (LAW) and 3rd Designing Meaning Representations (DMR) Workshop
}

Proceedings of the Workshop

November 11, 2021 
C2021 The Association for Computational Linguistics

Order copies of this and other ACL proceedings from:

Association for Computational Linguistics (ACL)

209 N. Eighth Street

Stroudsburg, PA 18360

USA

Tel: +1-570-476-8006

Fax: +1-570-476-0860

acleaclweb.org

$978-1-954085-85-5$ 


\section{Introduction to the Workshop}

Linguistic annotation of natural language corpora is the backbone of supervised methods of statistical natural language processing and has contributed to breakthroughs in practical natural language applications, most notably in Machine Translation, Machine Reading, Question Answering, and Recognizing Textual Entailment. Nonetheless, challenges remain in developing systems that can actually "understand" human language, including the ability to track entities in a text, understand the relations between these entities, track events and their participants described in a text, understand how events unfold in time, and distinguish events that have actually happened from events that are planned or intended, are uncertain, or did not happen at all. The fifteenth LAW and third DMR workshops have been combined for the first time to provide a forum for presentation and discussion of innovative research on all aspects of linguistic annotation, with a special focus on the design and annotation of representations of the various elements of meaning contributing to understanding. These proceedings include papers presented at LAW-DMR on November 11, 2021, held online in conjunction with the 2021 Conference on Empirical Methods in Natural Language Processing in Punta Cana, Dominican Republic.

This premier iteration of the joint LAW-DMR workshop received 35 submissions, out of which 18 papers have been accepted to be presented at the workshop -8 as talks and 10 as posters. In addition to oral and poster paper presentations, LAW-DMR also featured invited talks by Joyce Chai (University of Michigan) and Stephan Oepen (University of Oslo), and a panel session discussion of the topic, "What is the future of linguistic annotation?"

We thank the Association for Computational Linguistics' Special Interest Group for Annotation (ACL SIGANN), our organizing committee, for its continuing organization of the LAW workshops, and the EMNLP 2021 workshop chairs for their support. We are grateful to all of the authors for submitting their papers to the workshop and our program committee members for their dedication and their thoughtful reviews. Finally, we thank our invited speakers and panelists for making the workshop a uniquely valuable discussion of linguistic annotation research.

\section{Special Theme: Multi-layered and Multi-modal Annotations}

Given that this is the first joint LAW and DMR workshop, this year's theme was to explore multi-layered annotations, and how different types of annotations can be brought together to enhance progress towards true understanding. This includes papers on the broadening and deepening existing annotation schemas, as well as annotations going beyond text to mark up head movements. 



\section{Workshop Chairs}

Claire Bonial, DEVCOM U.S. Army Research Laboratory

Nianwen Xue, Brandeis University

\section{Organizing Committee}

Stefanie Dipper, Ruhr University Bochum

Annemarie Friedrich, Bosch Center for Artificial Intelligence

Chu-Ren Huang, The Hong Kong Polytechnic University

Nancy Ide, Vassar College

Lori Levin, Carnegie-Mellon University

Adam Meyers, New York University

Antonio Pareja-Lora, Universidad Complutense de Madrid / ATLAS, UNED

Massimo Poesio, University of Essex

Sameer Pradhan, University of Pennsylvania and cemantix.org

Ines Rehbein, Leibniz Science Campus, Institute for German Language and Heidelberg University

Manfred Stede, University of Potsdam

Katrin Tomanek, OpenTable

Fei Xia, University of Washington

Heike Zinsmeister, University of Hamburg

\section{Invited Speakers}

Joyce Chai, University of Michigan

Stephan Oepen, University of Oslo

\section{Program Committee}

Ron Artstein, USC Institute for Creative Technologies

Johan Bos, University of Groningen

Nicoletta Calzolari, ILC-CNR

Emmanuele Chersoni, Hong Kong Polytechnic University

Kathryn Conger, Universitiy of Colorado Boulder

William Croft, University of New Mexico

Valeria de Paiva, Topos Institute

Stefanie Dipper, Ruhr University Bochum

Lucia Donatelli, Saarland University

Jonathan Dunn, University of Canterbury

Kilian Evang, Heinrich Heine University Düsseldorf

Federico Fancellu, Samsung AI Research Canada

Annemarie Friedrich, Bosch Research

Kim Gerdes, Université Paris-Saclay

Jan Hajic, Charles University

Jena D. Hwang, Allen Institute for AI 
Nancy Ide, Vassar College

Elisabetta Jezek, Università di Pavia

Sandra Kübler, Indiana University

Lori Levin, Carnegie Mellon University

Bin Li, Nanjing Normal University

Adam Meyers, New York University

Kemal Oflazer, Carnegie Mellon University in Qatar

Simon Ostermann, Saarland University

Martha Palmer, University of Colorado

Antonio Pareja-Lora, Universidad de Alcalá de Henares

Massimo Poesio, Queen Mary University of London

Matt Post, Johns Hopkins University

Sameer Pradhan, University of Pennsylvania and cemantix.org

Weiguang QU, Nanjing Normal University

Ines Rehbein, University of Mannheim

Nathan Schneider, Georgetown University

Djamé Seddah, Inria

Manfred Stede, University of Potsdam

Zdenka Uresova, Charles University

Bonnie Webber, University of Edinburgh

Michael Wiegand, Alpen-Adria-Universitaet Klagenfurt

Hongzhi Xu, Shanghai International Studies University

Deniz Zeyrek, Middle East Technical University

Heike Zinsmeister, Universität Hamburg 


\section{Table of Contents}

Zero-shot cross-lingual Meaning Representation Transfer: Annotation of Hungarian using the Prague Functional Generative Description

Attila Novák, Borbála Novák and Csilla Novák

Theoretical and Practical Issues in the Semantic Annotation of Four Indigenous Languages

Jens E. L. Van Gysel, Meagan Vigus, Lukas Denk, Andrew Cowell, Rosa Vallejos, Tim O'Gorman

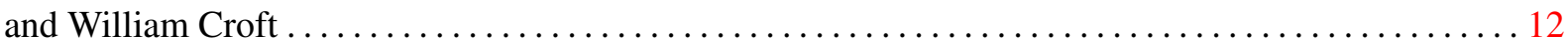

Representing Implicit Positive Meaning of Negated Statements in AMR

Katharina Stein and Lucia Donatelli ..................................... 23

AutoAspect: Automatic Annotation of Tense and Aspect for Uniform Meaning Representations

Daniel Chen, Martha Palmer and Meagan Vigus .............................. 36

Can predicate-argument relationships be extracted from UD trees?

Adam Ek, Jean-Philippe Bernardy and Stergios Chatzikyriakidis ................... 46

Classifying Divergences in Cross-lingual AMR Pairs

Shira Wein and Nathan Schneider ..................................... 56

A Linguistic Annotation Framework to Study Interactions in Multilingual Healthcare Conversational Forums

Ishani Mondal, Kalika Bali, Mohit Jain, Monojit Choudhury, Ashish Sharma, Evans Gitau, Jacki O’Neill, Kagonya Awori and Sarah Gitau ...................................... 66

Sister Help: Data Augmentation for Frame-Semantic Role Labeling

Ayush Pancholy, Miriam R L Petruck and Swabha Swayamdipta .................... 78

A Corpus Study of Creating Rule-Based Enhanced Universal Dependencies for German

Teresa Bürkle, Stefan Grünewald and Annemarie Friedrich ...................... 85

Subcategorizing Adverbials in Universal Conceptual Cognitive Annotation

Zhuxin Wang, Jakob Prange and Nathan Schneider............................. 96

Simplifying annotation of intersections in time normalization annotation: exploring syntactic and semantic validation

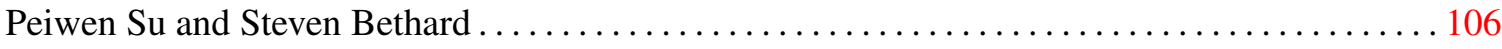

Overcoming the challenges in morphological annotation of Turkish in universal dependencies framework

Talha Bedir, Karahan Şahin, Onur Gungor, Suzan Uskudarli, Arzucan Özgür, Tunga Güngör and

Balkiz Ozturk Basaran ............................................... 112

Automatic Entity State Annotation using the VerbNet Semantic Parser

Ghazaleh Kazeminejad, Martha Palmer, Tao Li and Vivek Srikumar .................. 123

On Releasing Annotator-Level Labels and Information in Datasets

Vinodkumar Prabhakaran, Aida Mostafazadeh Davani and Mark Diaz................... 133

Increasing Sentence-Level Comprehension Through Text Classification of Epistemic Functions

Maria Berger and Elizabeth Goldstein ...................................... 139 
Towards a Methodology Supporting Semiautomatic Annotation of HeadMovements in Video-recorded Conversations

Patrizia Paggio, Costanza Navarretta, Bart Jongejan and Manex Agirrezabal . . 151

Intensionalizing Abstract Meaning Representations: Non-Veridicality and Scope

Gregor Williamson, Patrick Elliott and Yuxin Ji ............................. 160

WikiGUM: Exhaustive Entity Linking for Wikification in 12 Genres

Jessica Lin and Amir Zeldes ......................................... 170 


\section{Workshop Program}

Thursday, 11 November 2021

\section{8:45-09:00 Introduction}

09:00-11:00 Session A: Oral presentations:

09:00-10:00 Invited talk by Stephan Oepen: Linguistic Structure beyond Strings and Trees

10:00-10:15 Zero-shot cross-lingual Meaning Representation Transfer: Annotation of Hungarian using the Prague Functional Generative Description

Attila Novák, Borbála Novák and Csilla Novák

10:15-10:30 Theoretical and Practical Issues in the Semantic Annotation of Four Indigenous Languages

Jens E. L. Van Gysel, Meagan Vigus, Lukas Denk, Andrew Cowell, Rosa Vallejos, Tim O'Gorman and William Croft

10:30-10:45 Representing Implicit Positive Meaning of Negated Statements in AMR

Katharina Stein and Lucia Donatelli

10:45-11:00 AutoAspect: Automatic Annotation of Tense and Aspect for Uniform Meaning Representations

Daniel Chen, Martha Palmer and Meagan Vigus

\section{1:30-13:30 Session B: Poster presentations:}




\section{Thursday, 11 November 2021 (continued)}

\section{1:30-12:00 Overview of poster presentations}

12:00-12:45 Can predicate-argument relationships be extracted from UD trees?

Adam Ek, Jean-Philippe Bernardy and Stergios Chatzikyriakidis

12:00-12:45 Classifying Divergences in Cross-lingual AMR Pairs

Shira Wein and Nathan Schneider

12:00-12:45 A Linguistic Annotation Framework to Study Interactions in Multilingual Healthcare Conversational Forums

Ishani Mondal, Kalika Bali, Mohit Jain, Monojit Choudhury, Ashish Sharma, Evans Gitau, Jacki O’Neill, Kagonya Awori and Sarah Gitau

12:00-12:45 Sister Help: Data Augmentation for Frame-Semantic Role Labeling

Ayush Pancholy, Miriam R L Petruck and Swabha Swayamdipta

12:00-12:45 A Corpus Study of Creating Rule-Based Enhanced Universal Dependencies for German

Teresa Bürkle, Stefan Grünewald and Annemarie Friedrich

12:00-12:45 Subcategorizing Adverbials in Universal Conceptual Cognitive Annotation Zhuxin Wang, Jakob Prange and Nathan Schneider

12:00-12:45 Simplifying annotation of intersections in time normalization annotation: exploring syntactic and semantic validation

Peiwen Su and Steven Bethard

12:00-12:45 Overcoming the challenges in morphological annotation of Turkish in universal dependencies framework

Talha Bedir, Karahan Şahin, Onur Gungor, Suzan Uskudarli, Arzucan Özgür, Tunga Güngör and Balkiz Ozturk Basaran

12:00-12:45 Automatic Entity State Annotation using the VerbNet Semantic Parser Ghazaleh Kazeminejad, Martha Palmer, Tao Li and Vivek Srikumar

12:00-12:45 On Releasing Annotator-Level Labels and Information in Datasets Vinodkumar Prabhakaran, Aida Mostafazadeh Davani and Mark Diaz 
Thursday, 11 November 2021 (continued)

12:45-13:30 Panel discussion: what's the future of linguistic annotation?

14:00-16:00 Session C: Oral presentations:

14:00-15:00 Invited talk by Joyce Chai: Intuitive Physics in Commonsense Language Understanding

15:00-15:15 Increasing Sentence-Level Comprehension Through Text Classification of Epistemic Functions

Maria Berger and Elizabeth Goldstein

15:15-15:30 Towards a Methodology Supporting Semiautomatic Annotation of HeadMovements in Video-recorded Conversations

Patrizia Paggio, Costanza Navarretta, Bart Jongejan and Manex Agirrezabal

15:30-15:45 Intensionalizing Abstract Meaning Representations: Non-Veridicality and Scope Gregor Williamson, Patrick Elliott and Yuxin Ji

15:45-16:00 WikiGUM: Exhaustive Entity Linking for Wikification in 12 Genres Jessica Lin and Amir Zeldes

\section{6:00-16:15 Concluding remarks}


